\title{
2D-Mueller-matrix tomography of optically anisotropic polycrystalline networks of biological tissues histological sections
}

\author{
L. Trifonyuk ${ }^{\mathrm{a}}$, W. Baranowski ${ }^{\mathrm{b}}$, V. Ushenko ${ }^{\mathrm{c}}, \mathrm{O}^{\mathrm{O}}$ Olar $^{\mathrm{c}}$, A. Dubolazov ${ }^{\mathrm{c}, *}$, \\ Yu. Ushenko ${ }^{c}$, B. Bodnar ${ }^{d}$, O. Vanchulyak ${ }^{d}$, L. Kushnerik ${ }^{c}$, M. Sakhnovskiy ${ }^{c}$ \\ a Rivne State Medical Center, 78 Kyivska Str., Rivne, 33007, Ukraine \\ ${ }^{\mathrm{b}}$ Warsaw Military Institute of Medicine, ul. Szaserów 128, Warsaw, 04141, Poland \\ c Chernivtsi National University, 2 Kotsiubynskyi Str., Chernivtsi, 58012, Ukraine \\ d Bukovinian State Medical University, 3 Theatral Sq., Chernivtsi, 58000, Ukraine
}

\section{A R T I C L E I N F O}

\section{Article history:}

Received 23 August 2017

Received in revised form 5 June 2018

Accepted 11 July 2018

\section{Keywords:}

Polarization

Stokes-polarimetry

Mueller matrix

Tomography, differential matrix

Birefringence

Dichroism

Biological tissues

\begin{abstract}
A B S T R A C T
A new technique of Mueller-matrix mapping of the birefringent structure of biological preparations of human organs tissues is suggested. The algorithms of reconstruction of average values and magnitude of fluctuations of the phase (birefringence) and amplitude (dichroism) of optically anisotropic structure of myocardium and connective tissue component of the vaginal wall histological section are proposed. The magnitudes and ranges of changes in the statistical moments of the 1st-4th order that characterize the distribution of average values and magnitude of fluctuations of birefringence and dichroism of the myocardium and connective tissue of the vaginal tissues histological sections were determined. Joint studies of distributions of the characteristics of phase and amplitude of the anisotropy of myocardium and connective tissue component of the vaginal wall tissues of different states were performed. The cases of various necrotic changes in the myocardium and pathological conditions of the vagina wall (prolapse of the genitals) are examined. Balanced accuracy of the method of Mueller-matrix polarization-phase and diffuse tomography of optically anisotropic polycrystalline networks in the differentiation of necrotic and pathological changes in human organs is determined.
\end{abstract}

(C) 2018 Association of Polish Electrical Engineers (SEP). Published by Elsevier B.V. All rights reserved.

\section{Introduction}

One of the mostcommon directions in the analysis of mechanisms of conversion of optical radiation by biological layers is the statistical [1-4] and correlation optical [5-8] approach. Important place in different directions of diagnostics of light-scattering biological layers structure belongs to polarization and Mueller-matrix techniques [9-12]. Sufficient progress is achieved in polarimetric studies of optically thin (non-depolarizing) biological layers. Here, based on the statistical approach, correlations are found between the average, dispersion, skewness, and kurtosis, which characterize the phase-inhomogeneous layers and Mueller-matrix elements of the distributions [13-15]. On this basis, diagnostic criteria for cancer and other pathologies of human organs were found [16-19]. However, real biological media usually have depolarizing effect on the laser beam, propagating in them [20-23]. Because of this, the

\footnotetext{
* Corresponding author.

E-mail address: a.dubolazov@chnu.edu.ua (A. Dubolazov).
}

unilateral relationship between the parameters of biological object and the distribution of matrix elements is broken. Thus, development of new methods based on a differentiation of fully polarized and completely depolarized Mueller-matrix components is important [24-26].

In our work, we consider the possibility of expanding functionality of the methods of matrix mapping and $2 \mathrm{D}$ reconstruction of distributions of average values and magnitude of fluctuations of birefringence and dichroism in the differentiation of necrotic (myocardium) and pathological (prolapse of the genitals) changes of biological tissues.

\section{Theory}

It is known that biological tissues have a complex anisotropy which is manifested in linear (fibrillar molecular structures) and circular (chirality of the molecular complexes structure) birefringence mechanisms [1,13-15,27,28].

Theoretical background of the Mueller matrix approach to describe the interaction of optical radiation with depolarizing lay- 
ers is shown in a series of publications [20-23]. This dependence is analytically illustrated by the equation:

$\frac{d\|B\|}{d z}=\|B(z)\|\|D(z)\|$

where $\|B(z)\|$ is the Mueller matrix of the object in the plane $z$ and $\|D(z)\|$ is the differential matrix operator.

For optically thin layers, which do not depolarize, but transform the polarization of the probing beam, the matrix operator $\|D(z)\|$ consists of six parameters that completely describe phase and amplitude anisotropy of the biological layer:

$\|D\|=\left\|\begin{array}{cccc}0 & \Delta_{0,90} & \Delta_{45,135} & \Delta_{\otimes, \oplus} \\ \Delta_{0,90} & 0 & \Phi_{\otimes, \oplus} & -\Phi_{45,135} \\ \Delta_{45,135} & -\Phi_{\otimes, \oplus} & 0 & \Phi_{0,90} \\ \Delta_{\otimes, \oplus} & \Phi_{45,135} & -\Phi_{0,90} & 0\end{array}\right\|$

where the parameters are defined as:

- $\Delta_{0,90}, \Delta_{45,135}$ - linear dichroism;

- $\Phi_{0,90}, \Phi_{45,135}$ - linear birefringence for orthogonal components $0^{0}-90^{0}$ and $45^{0}-135^{0}$;

- $\Delta_{\otimes, \oplus}$ and $\Phi_{\otimes, \oplus}$ - circular dichroism and birefringence for right$(\otimes)$ and left- $(\oplus)$ circularly polarized components.

Then, the parameters of phase and amplitude anisotropy are determined by the following relations:

$$
\begin{aligned}
& \Phi_{0,90} \equiv \delta_{0,90}=\frac{2 \pi}{\lambda} \Delta n_{0,90} l ; \quad \Delta n_{0,90}=n_{0}-n_{90} ; \\
& \Phi_{45 ; 135} \equiv \delta_{45 ; 135}=\frac{2 \pi}{\lambda} \Delta n_{45 ; 135} l ; \quad \Delta n_{45 ; 135}=n_{45}-n_{135} ; \\
& \Phi_{\otimes, \oplus} \equiv \phi_{\otimes, \oplus}=\frac{2 \pi}{\lambda} \Delta n_{\otimes, \oplus} l ; \quad \Delta n_{\otimes, \oplus}=n_{\otimes}-n_{\oplus} \\
& \Delta_{0,90} \equiv \tau_{0 ; 90}=\frac{2 \pi}{\lambda} \Delta \tau_{0 ; 90} l ; \quad \Delta \tau_{0,90}=\tau_{0}-\tau_{90} ; \\
& \Delta_{45 ; 135} \equiv \tau_{45 ; 135}=\frac{2 \pi}{\lambda} \Delta \tau_{45 ; 135} l ; \quad \Delta \tau_{45 ; 135}=\tau_{45}-\tau_{135} ; \\
& \Delta_{\otimes ; \oplus} \equiv \chi_{\otimes ; \oplus}=\frac{2 \pi}{\lambda} \Delta \tau_{\otimes ; \oplus} l ; \quad \Delta \tau_{\otimes ; \oplus}=\tau_{\otimes}-\tau_{\oplus} .
\end{aligned}
$$

Here $\delta_{0 ; 90}$ and $\delta_{45 ; 135}$ are the phase shifts of linear birefringence between the orthogonal $\left(0^{0}-90^{0}, 45^{0}-135^{0}\right)$ linearly polarized components of laser wave amplitude; $\phi_{\otimes ; \oplus}$ is the phase shift of circular birefringence between the orthogonal (right $\otimes$ and left $\oplus$ ) circularly polarized components of laser wave amplitude; $\tau_{0 ; 90}$ and $\tau_{45 ; 135}$ are the phase shifts of linear dichroism between the orthogonal $\left(0^{0}-90^{0}, 45^{0}-135^{0}\right)$ linearly polarized components of laser wave amplitude; $\chi_{\otimes ; \oplus}$ is the is the phase shift of circular dichroism between the orthogonal (right $\otimes$ and left $\oplus$ ) circularly polarized components of laser wave amplitude; $\Delta n_{0 ; 90}$ and $\Delta n_{45 ; 135}$ are the values of linear birefringence; $\Delta n_{\otimes ; \oplus}$ is the value of circular birefringence; $\Delta \tau_{0 ; 90}$ and $\Delta \tau_{45 ; 135}$ are the values of linear dichroism; $\Delta \tau_{\otimes ; \oplus}$ is the value of circular dichroism; $n_{j}$ and $\tau_{j}$ are the refraction and absorption indices for the orthogonal polarized components $\left(0^{0}-90^{\circ}, 45^{0}-135^{0}\right.$ and $\left.\otimes-\oplus\right)$ of the laser radiation amplitude; $l$ is the geometrical thickness; $\lambda$ is the laser beam wavelength.

Further (without reducing the completeness of the analysis), according to Refs. [20] and [21], we consider generalized parameters of the linear birefringence $(\Phi)$ and dichroism $(\Delta)$ :

$\Phi=\sqrt{\Phi_{0 ; 90}^{2}+\Phi_{45 ; 135}^{2}}$
$\Delta=\sqrt{\Delta_{0 ; 90}^{2}+\Delta_{45 ; 135 .}^{2}}$
For a depolarizing medium the matrix operator $\langle\||D(z)| \mid\rangle$ in expression (1) can be represented as average (polarized part of $\| D(z)||)$ and fluctuating $\|\tilde{D}(z)\|$ (depolarized part of $\|D(z)\|$ ) components giving:

$\|D(z)\|=L T ;\|D(z)\|>+\|\tilde{D}(z)\|$.

It should be noted that there always is the feedback:

$\| B(z)||=\exp (\| D(z)||)$.

The simultaneous analysis of Eqs. (1), (2), (11), (12) enabled to obtain the expression of the logarithmic matrix algorithm:

$\Lambda(z)=\ln \{\|B(z)\|\}=P(z)+H(z)$

that is determined as a superposition of antisymmetric $P(z)$ (polarization) and symmetric $H(z)$ (depolarization) components $\Lambda(z)$ according to:

$P(z)=\langle\|D\|\rangle z ; \quad H(z)=0,5\|\tilde{D}\| z^{2}$,

where

$$
\begin{aligned}
& P=0.5\left(\Lambda-M \Lambda^{T} M\right) ; \quad H=0.5\left(\Lambda+M \Lambda^{T} M\right) ; \\
& M=\operatorname{diag}(1,-1,-1,-1) .
\end{aligned}
$$

Here $M$ is the metric Minkovsky matrix [24-26].

Let's consider the algorithms for determining the antisymmetric $P(z)$ (polarization) and symmetric $H(z)$ (depolarization) components of $\Lambda(z)$.

\subsection{Polarization $P(z)$ component}

Taking into account Eqs. (2), (13-15), the polarization component of the matrix logarithmic algorithm takes the form:

$$
\langle\|D\|\rangle=z^{-1}\left\|\begin{array}{cccc}
0 & \left(l_{12}+l_{21}\right) & \left(l_{13}+l_{31}\right) & \left(l_{14}+l_{41}\right) \\
\left(l_{21}+l_{12}\right) & 0 & \left(l_{23}-l_{32}\right) & \left(l_{24}-l_{42}\right) \\
\left(l_{31}+l_{13}\right) & \left(l_{32}-l_{23}\right) & 0 & \left(l_{34}-l_{43}\right) \\
\left(l_{41}+l_{14}\right) & \left(l_{42}-l_{24}\right) & \left(l_{43}-l_{34}\right) & 0
\end{array}\right\|
$$

where

$$
\left\{\begin{array}{l}
l_{i k}=\ln B_{i k} \\
l_{i k}+l_{k i}=\ln \left(B_{i k} \times B_{k i}\right) \\
l_{i k}-l_{k i}=\ln \left(\frac{B_{i k}}{B_{k i}}\right)
\end{array}\right.
$$

Taking into account relations (15), the non-zero elements of the matrix $\langle\|D\|\rangle$ Eq. (16) makes it possible to obtain:

$$
\langle\|D\|\rangle=z^{-1}\left\{\begin{array}{l}
\left\langle d_{12}\right\rangle=\left\langle d_{21}\right\rangle=\ln \left(B_{12} B_{21}\right) ; \\
\left\langle d_{13}\right\rangle=\left\langle d_{31}\right\rangle=\ln \left(B_{13} B_{31}\right) ; \\
\left\langle d_{14}\right\rangle=\left\langle d_{41}\right\rangle=\ln \left(B_{14} B_{41}\right) ; \\
\left\langle d_{23}\right\rangle=-\left\langle d_{32}\right\rangle=\ln \left(\frac{B_{23}}{B_{32}}\right) ; \\
\left\langle d_{24}\right\rangle=-\left\langle d_{42}\right\rangle=\ln \left(\frac{B_{24}}{B_{42}}\right) ; \\
\left\langle d_{34}\right\rangle=-\left\langle d_{43}\right\rangle=\ln \left(\frac{B_{34}}{B_{43}}\right) .
\end{array}\right.
$$

The common analysis of relations (3)-(8) and (18) allows obtaining the algorithms of polarization-phase tomography as analytical 
layer-by-layer $(0 \leq z \leq l)$ expressions of Mueller matrix with the step $\Delta z$ reproduction of average values of the parameters $\delta ; \phi$, $\tau ; \quad \chi$ giving the optical anisotropy of biological layers:

$\delta_{0,90}=\frac{2 \pi z}{\lambda} \Delta n_{0,90}=\ln \left(\frac{B_{24}}{B_{42}}\right)$

$\delta_{45,135}=\frac{2 \pi z}{\lambda} \Delta n_{45,135}=\ln \left(\frac{B_{34}}{B_{43}}\right)$

$\phi_{\otimes, \oplus}=\frac{2 \pi z}{\lambda} \Delta n_{\otimes, \oplus}=\ln \left(\frac{B_{23}}{B_{32}}\right)$

$\tau_{0,90}=\frac{2 \pi z}{\lambda} \Delta \tau_{0 ; 90}=\ln \left(B_{12} B_{21}\right)$

$\tau_{45,135}=\frac{2 \pi z}{\lambda} \Delta \tau_{45 ; 135}=\ln \left(B_{13} B_{31}\right)$

$\chi_{\otimes, \oplus}=\frac{2 \pi z}{\lambda} \Delta \tau_{\otimes, \oplus}=\ln \left(B_{14} B_{41}\right)$.

Using the ideologies of differential data analysis of Muellermatrix mapping allowed us to obtain a set of algorithms [Eqs. properties $\xi_{i=1-6}$ [ratio (2) - (8)] of the scattering medium is considered as the average $\mu_{i}$ and the fluctuating $\sigma_{i}$ components:

$\xi_{i}=\mu_{i}+\sigma_{i}$

Here

$\mu_{i}=\left(\begin{array}{l}\mu_{1}=\left\langle\Delta_{0 ; 90}\right\rangle ; \\ \mu_{2}=\left\langle\Delta_{45 ; 135}\right\rangle ; \\ \mu_{3}=\left\langle\Delta_{\otimes ; \oplus}\right\rangle ; \\ \mu_{4}=\left\langle\Phi_{0 ; 90}\right\rangle ; \\ \mu_{5}=\left\langle\Phi_{45 ; 135}\right\rangle ; \\ \mu_{6}=\left\langle\Phi_{\otimes ; \oplus}\right\rangle ;\end{array}\right) ; \sigma_{i}=\left(\begin{array}{l}\sigma_{1}=\sqrt{\theta_{1}\left(\Delta_{0 ; 90}\right)} \\ \sigma_{2}=\sqrt{\theta_{2}\left(\Delta_{45 ; 135}\right)} \\ \sigma_{3}=\sqrt{\theta_{3}\left(\Delta_{\otimes ; \oplus}\right)} ; \\ \sigma_{4}=\sqrt{\theta_{4}\left(\Phi_{0 ; 90}\right)} \\ \sigma_{5}=\sqrt{\theta_{5}\left(\Phi_{45 ; 135}\right)} \\ \sigma_{6}=\sqrt{\theta_{6}\left(\Phi_{\otimes ; \oplus}\right)} .\end{array}\right)$

where $\sqrt{\theta_{i}}$ is the standard deviation of fluctuations of the polarization properties $\xi_{i=1-6}$.

In the approximation of Eqs. (27) and (28) V. Devlaminck [22-24] got the next expression for the differential matrix of the $2^{\text {nd }}$ order:

$$
\begin{aligned}
& \|\tilde{D}\|= \\
& =\left\|\begin{array}{cccc}
\left(\sigma_{4}^{2}+\sigma_{5}^{2}+\sigma_{6}^{2}\right)_{11} & -0,5\left(\sigma_{2} \sigma_{6}-\sigma_{3} \sigma_{5}\right)_{12} & -0,5\left(\sigma_{3} \sigma_{4}-\sigma_{1} \sigma_{6}\right)_{13} & -0,5\left(\sigma_{1} \sigma_{5}-\sigma_{2} \sigma_{4}\right)_{14} \\
0,5\left(\sigma_{2} \sigma_{6}-\sigma_{3} \sigma_{5}\right)_{21} & \left(\sigma_{4}^{2}-\sigma_{2}^{2}-\sigma_{3}^{2}\right)_{22} & 0,5\left(\sigma_{1} \sigma_{2}+\sigma_{4} \sigma_{5}\right)_{23} & 0,5\left(\sigma_{1} \sigma_{3}+\sigma_{4} \sigma_{5}\right)_{24} \\
0,5\left(\sigma_{3} \sigma_{4}-\sigma_{1} \sigma_{6}\right)_{31} & 0,5\left(\sigma_{1} \sigma_{2}+\sigma_{4} \sigma_{5}\right)_{32} & \left(\sigma_{5}^{2}-\sigma_{1}^{2}-\sigma_{3}^{2}\right)_{33} & 0,5\left(\sigma_{2} \sigma_{3}+\sigma_{5} \sigma_{6}\right)_{34} \\
0,5\left(\sigma_{1} \sigma_{5}-\sigma_{2} \sigma_{4}\right)_{41} & 0,5\left(\sigma_{1} \sigma_{3}+\sigma_{4} \sigma_{6}\right)_{42} & 0,5\left(\sigma_{2} \sigma_{3}+\sigma_{5} \sigma_{6}\right)_{43} & \left(\sigma_{6}^{2}-\sigma_{1}^{2}-\sigma_{3}^{2}\right)_{44}
\end{array}\right\|
\end{aligned}
$$

(19)-(24)] of polarization reproduction of average values of birefringence and dichroism of biological layers.

\subsection{Depolarization $H(z)$ component}

It can be shown that the symmetric component $H\left(\tilde{D}_{i k}\right)$ (ratio (13)) is determined by the matrix operator of the next symmetry:

$\|\tilde{D}\|=0,5 z^{-2}\left\|\begin{array}{cccc}l_{11} & \left(l_{12}-l_{21}\right) & \left(l_{13}-l_{31}\right) & \left(l_{14}-l_{41}\right) \\ \left(l_{21}-l_{12}\right) & l_{22} & \left(l_{23}+l_{32}\right) & \left(l_{24}+l_{42}\right) \\ \left(l_{31}-l_{13}\right) & \left(l_{32}+l_{23}\right) & l_{33} & \left(l_{34}+l_{43}\right) \\ \left(l_{41}-l_{14}\right) & \left(l_{42}+l_{24}\right) & \left(l_{43}+l_{34}\right) & l_{44}\end{array}\right\|$

In expanded form (25) is written as follows:

$$
\|\tilde{D}\|=0,5 z^{-2}\left\|\begin{array}{cccc}
\ln B_{11} & \ln \left(\frac{B_{12}}{B_{21}}\right) & \ln \left(\frac{B_{13}}{B_{31}}\right) & \ln \left(\frac{B_{14}}{B_{41}}\right) \\
\ln \left(\frac{B_{21}}{B_{12}}\right) & \ln B_{22} & \ln \left(B_{23} B_{32}\right) & \ln \left(B_{24} B_{42}\right) \\
\ln \left(\frac{B_{31}}{B_{13}}\right) & \ln \left(B_{32} B_{23}\right) & \ln B_{33} & \ln \left(B_{34} B_{43}\right) \\
\ln \left(\frac{B_{41}}{B_{14}}\right) & \ln \left(B_{42} B_{24}\right) & \ln \left(B_{43} B_{34}\right) & \ln B_{44}
\end{array}\right\|
$$

The theoretical analysis of the MMI of the depolarization component $\tilde{D}_{i k}$ is based on our generalization of V. Devlaminck's theory $[22-24]$. Here, the value of each of the elementary polarization
The analysis of Eq. (29) reveals the following physical content of the partial elements of the depolarization component of an optically thick biological layer Mueller matrix with fluctuations of the linear and circular birefringence and dichroism parameters:

- diagonal elements $\tilde{d}_{11 ; 22 ; 33 ; 44}$ are determined by combinations of dispersion $\theta_{i=1 \div 6}$ of the parameters fluctuations of various mechanisms of phase $\left(\theta_{4 \div 6}\right)$ and amplitude $\left(\theta_{1 \div 3}\right)$ anisotropy;

- off-diagonal elements $d_{i k ; i \neq k}$ characterize the degree of mutual correlation of fluctuations in the parameters of linear and circular birefringence $\left(\sigma_{4 \div 6}\right)$ and dichroism $\left(\sigma_{1 \div 3}\right)$.

It was shown in Refs. $[25,26]$ that at the increase of light scattering multiplicity, a second-order differential matrix $\|\tilde{D}\|$ tends to a diagonal matrix $\operatorname{diag}\left(\tilde{d}_{11} ; \tilde{d}_{22} ; \tilde{d}_{33} ; \tilde{d}_{44}\right)$. Therefore, in our work we confine ourselves to the analysis of coordinate distributions:

$\tilde{T}\left(\tilde{D}_{i i}\right)=0.5 z^{-2}\left\{\begin{array}{l}\tilde{d}_{11}=\left(\theta_{4}+\theta_{5}+\theta_{6}\right)=\ln \left(B_{11}\right) ; \\ \tilde{d}_{11}=\left(\theta_{4}-\theta_{2}-\theta_{3}\right)=\ln \left(B_{22}\right) ; \\ \tilde{d}_{11}=\left(\theta_{5}-\theta_{1}-\theta_{3}\right)=\ln \left(B_{33}\right) ; \\ \tilde{d}_{11}=\left(\theta_{6}-\theta_{1}-\theta_{2}\right)=\ln \left(B_{44}\right) .\end{array}\right\}$

On the basis of our theoretical consideration, we propose the following principles of 2D Mueller-matrix tomography of histological sections of depolarizing biological tissues:

- Stockes-polarimetric mapping (according to the traditional method presented in the reviews [13-19]) of biological preparations and the definition of a series of Mueller-matrix images $B_{i k}(x, y)$; 


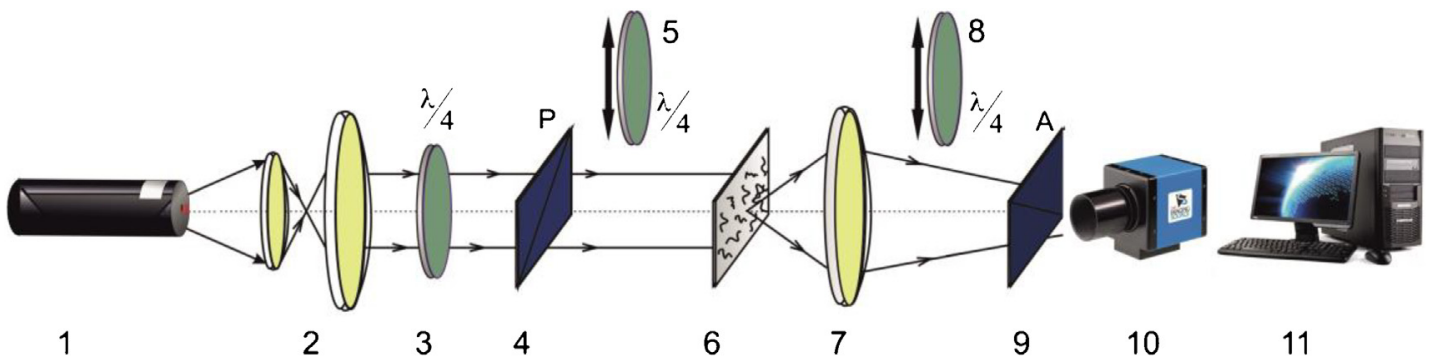

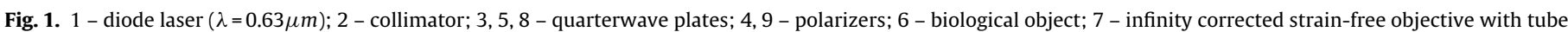
lens; 10 - digital camera; 11 - PC.

- Obtaining the polarization-phase tomograms - calculation [Eqs. (18) and (19)-(24)] of coordinate distributions $T\left(\left\langle d_{i k}\right\rangle\right)=$ $\left\{\begin{array}{l}\delta \\ \phi \\ \tau \\ \chi\end{array}\right\}(x, y)$

- Obtaining diffuse tomograms - calculation (Eqs. (26) and (27)-(30)] of coordinate distributions $\tilde{T}\left(\tilde{d}_{i i}\right)=$ $\left(\theta_{4}+\theta_{5}+\theta_{6}\right)^{-1}\left\{\begin{array}{c}1 \\ \left(\theta_{4}-\theta_{2}-\theta_{3}\right) ; \\ \left(\theta_{5}-\theta_{1}-\theta_{3}\right) ; \\ \left.\theta_{6}-\theta_{1}-\theta_{2}\right)\end{array}\right\}(x, y)$

- Statistical (statistical moments of the 1st-4th order [13]) and information (balanced accuracy of the method [15]) analysis of the distributions $T(x, y)$ and $\tilde{T}(x, y)$.

\section{Materials and methods}

Experimental study of the 2D structure of Mueller matrix ||$B \mid$ was performed in the polarimeter shown in Fig. 1. The devices, as well as the parameters and measurement procedures are presented in Refs. [16], [17], [27] and [28]. Here, we only give the optical scheme of the polarimeter for better understanding of the process of our study.

\section{Results and discussion}

This part of the work presents detailed experimental and statistical results of Mueller-matrix tomography in the expert evaluation of degenerative-dystrophic changes in myocardial tissue, as well as the accuracy of the clinical application of this method in the differential diagnosis of the connective tissue component of the vaginal wall during genital prolapse.

\subsection{Samples preparation}

The histological sections (geometrical thickness $d=30 \mu \mathrm{m} \div 40 \mu \mathrm{m})$ for the biopsy of myocardium and connective tissue component of the vaginal wall tissues were prepared using conventional freezing microtome. In this case such standard samples appear to partly depolarize (attenuation coefficient $\tau=0.05 \div 0.07$, degree of depolarization $\Lambda=35 \% \div 42 \%$ ) laser radiation. Therefore, in order to describe such samples it is not enough to use traditional for laser polarimetry approximation of non-depolarizing optically thin layers ( $\tau<0.01$ ) [13-15,29-31].

Further generalization of Mueller-matrix mapping of histological sections of biological tissues requires consideration as polarization $\langle\| D(z)||\rangle(18)$, as depolarization $\|\tilde{D}(z)\|$ (26) parts of matrix operator.

We investigated the samples of biological tissues with two types of polycrystalline structure changes:
- Degenerative-dystrophic in the post-mortem period or n̈ecrotic;

- Pathological; due to the presence of the disease.

As specific examples of such changes it were considered:

- necrotic changes - histological sections of myocardial biopsy of the patients who died of acute coronary insufficiency (type A, 36 samples) and ischemic disease (type B, 36 samples);

- pathological changes - of the healthy patients (type C, 33 samples); of those suffering from genitals prolapse (type D, 33 samples).

A collection of samples of type A and type C - test groups; of type B and type D - groups to be diagnosed.

\subsection{Polarization-phase Mueller-matrix tomography of myocardium histological sections}

The data of the polarization-phase Mueller-matrix tomography of the distributions $T\left(\left\langle d_{i k}\right\rangle\right)=\left\{\begin{array}{l}\delta \\ \phi \\ \tau \\ \chi\end{array}\right\}(x, y)$ obtained for the histological sections of the myocardium are presented in the series of Figs. 2 and 3.

Quantitative estimation of the optically anisotropic structure of biological preparations was carried out through calculations by the known algorithms [32] of a set of central statistical moments $Z_{i=1 ; 2 ; 3 ; 4}$ that characterize the average $\left(Z_{1}\right)$, dispersion $\left(Z_{2}\right)$, skewness $\left(Z_{3}\right)$ and kurtosis $\left(Z_{4}\right)$ characterizing the distribution of a series of polarization-phase tomograms $[T(x, y)]$.

The analysis of distribution of the parameters values $T\left(\left\langle d_{i k}\right\rangle\right)=$ $\left\{\begin{array}{l}\delta \\ \phi \\ \tau \\ \chi\end{array}\right\}(x, y)$ showed that the myocardial samples of both groups of the dead patients are characterized by all kinds of linear and circular birefringence and dichroism. The linear birefringence (fragments [(1),(2),(5),(6), Fig. 2)] is more vivid in comparison with the mechanisms of circular phase (fragments [(3),(4),(7),(8), Fig. 2] and amplitude (fragments [(1)-(8), Fig. 3] anisotropy.

The comparison of the polarization phase of myocardial tomograms of the type A and type B samples has revealed a higher level of optical anisotropy in type B.

The predominance $\left(\left\{\begin{array}{l}\langle\delta\rangle>\langle\tau\rangle ; \\ \phi\rangle>\langle\chi\rangle\end{array}\right\}\right)$ of the mechanisms of phase anisotropy of fibrillar myocardial networks over manifestations of dichroism can be explained by the minimal absorption in the red $\ddot{(} \lambda=0.63 \mu \mathrm{m})$ region of the protein molecules of myosin and actin. The spectral absorption maximum of these compounds falls on the ultraviolet region of the spectrum $(\Delta \lambda=250 \mu \mathrm{m} \div 350 \mu \mathrm{m})$ [19]. 


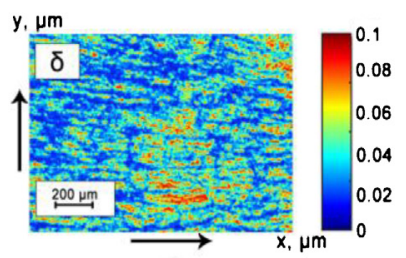

(1)

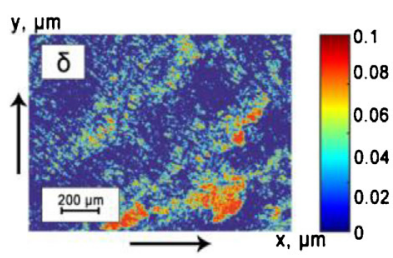

(5)
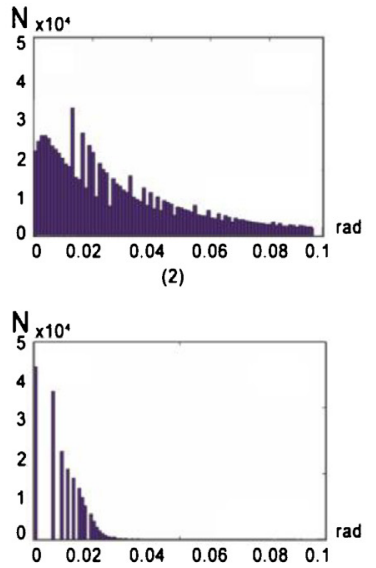

(6)

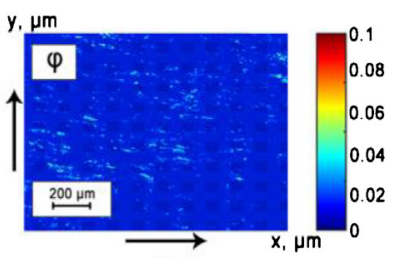

(3)

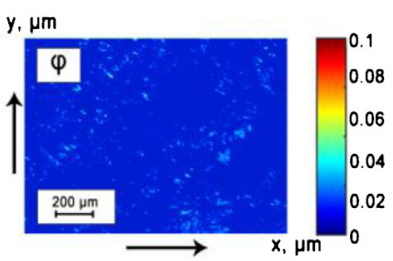

(7)

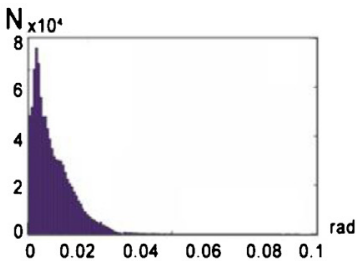

(4)

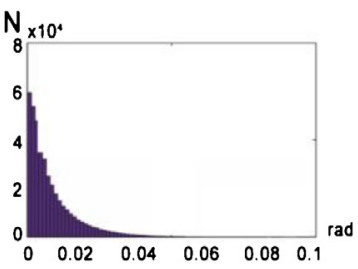

(8)

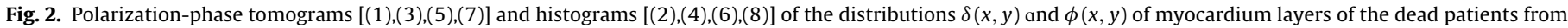
type A [(1) - (4)] and type B [(5) - (8)].

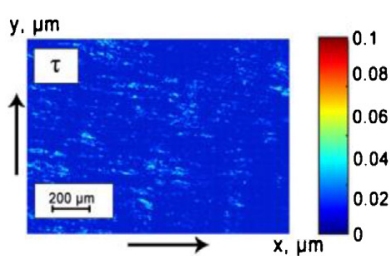

(1)

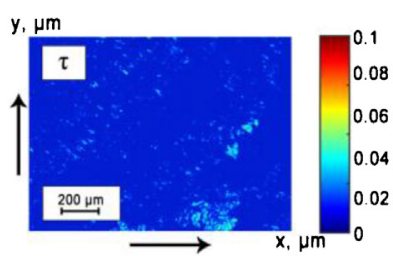

(5)

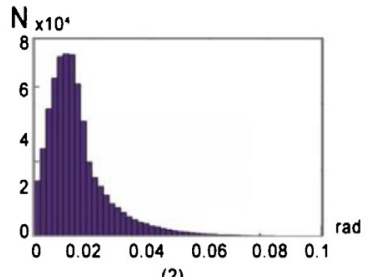

(2)

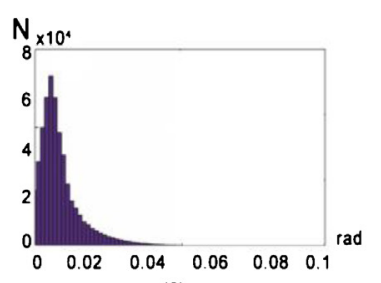

(6)

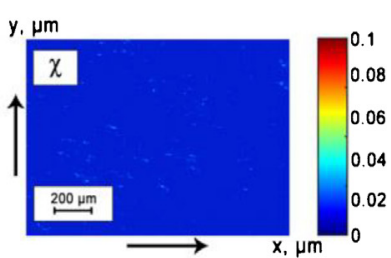

(3)

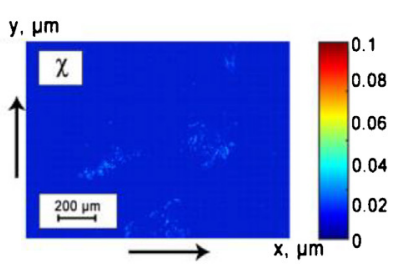

(7)
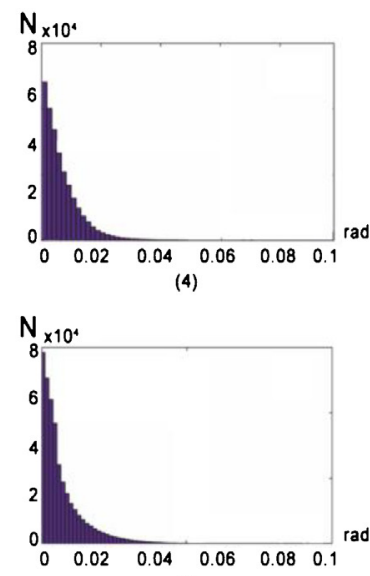

(8)

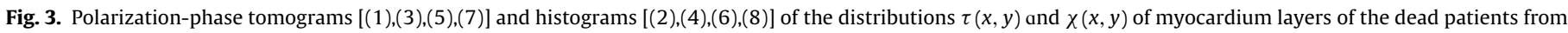
type A [(1) - (4)] and type B [(5) - (8)].

Long process of ischemic heart disease leads to degenerative and dystrophic changes of myocardium. Optically it is manifested in the decrease of mechanisms of structural anisotropy (linear birefringence and, to a lesser extent, dichroism) due to disorientation and reduction of geometric dimensions of myosin fibrils [13,19]. Therefore, the distribution histograms of phase shifts $N(\delta)$ [fragments (2),(6), Fig. 2] and the absorption coefficient $N(\tau)$ [fragments (2),(6), Fig. 3] obtained for histological sections of type B myocardium are characterized by lower values of the central moments of the $1 \mathrm{st}$ and 2 nd orders.

Similar parameters of the 3rd and 4th orders, on the contrary, increase. In order to obtain quantitative estimates of differentiation of myocardial samples with different pathology the statistical analysis of the structure of tomograms $T(x, y)$ within both groups was performed.

\subsection{Diffuse Mueller-matrix tomography of myocardium histological sections}

In this part of the research, we will analyze the results of the diffuse Mueller-matrix tomography method of depolarizing histological sections of the myocardium under the assumption $\left\{\begin{array}{l}\langle\delta\rangle>\langle\tau\rangle ; \\ \langle\phi\rangle>\langle\chi\rangle\end{array}\right\}$. Proceeding from this, the structure of the experimentally obtained distributions $\left\{\begin{array}{ll} & \tilde{T} \\ \tilde{T} & \left.\tilde{d}_{22}\right) \\ \tilde{T} & \left.\tilde{d}_{33}\right) \\ \tilde{T} & \left(\tilde{d}_{44}\right)\end{array}\right\}(x, y)$ is mainly determined by fluctuations (Eqs. (28) and (29)] of the parameters of linear $\left[\theta_{4}\left(\Phi_{0 ; 90}\right)\right.$ and $\left.\theta_{5}\left(\Phi_{45 ; 135}\right)\right]$ and circular birefringence $\left[\theta_{6}\left(\Phi_{\otimes ; \oplus}\right)\right]$.

Therefore, as it follows from Refs. [20] and [21], similarly to the case of polarization-phase tomography [Eq. (9)], we shall consider the generalized parameters of fluctuations of linear $\left(\theta_{\delta}\right)$ and circular $\left(\theta_{\phi}\right)$ birefringence:

$\left\{\begin{array}{l}\theta_{\delta}=\sqrt{\theta_{4}^{2}+\theta_{5}^{2}}=\sim_{d_{11}}^{-1} \sqrt{\tilde{d}_{22}^{2}+\tilde{d}_{33}^{2}} . \\ \theta_{\phi} \equiv \theta_{6}=\tilde{d}_{11}^{-1} d .44\end{array}\right.$

The data of diffuse Mueller-matrix tomography $\left(\tilde{T}\left(\theta_{\delta}\right)\right.$ and $\left.\tilde{T}\left(\theta_{\phi}\right)\right)$ scattering samples of the myocardium are presented in Fig. 4 .

The comparative analysis of the maps $\theta_{\delta}(x, y), \theta_{\phi}(x, y)$ and histograms $N\left(\theta_{\phi}\right), N\left(\theta_{\phi}\right)$ depolarizing the histological sections of the myocardium [Fig. 4,(1)-(8)] revealed a smaller level of fluctuations in the values of $\theta_{\delta}$ and $\theta_{\phi}$, like in the case of studies of polarizationphase tomograms [Fig. 2, type B layers with necrotic destruction of birefringent fibrillar networks. Quantitatively this fact illustrates 


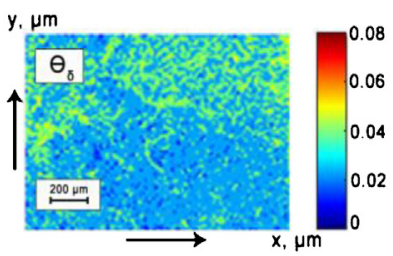

(1)

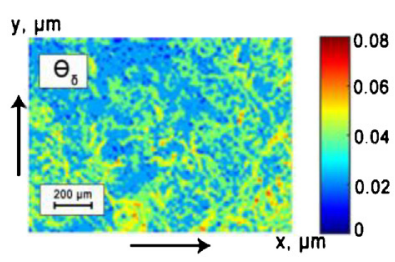

(5)

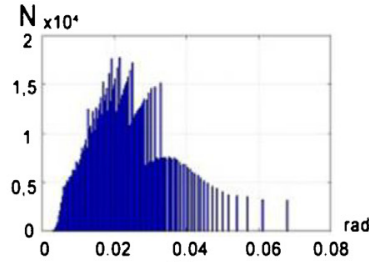

(2)

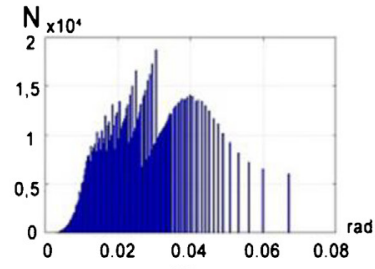

(6)

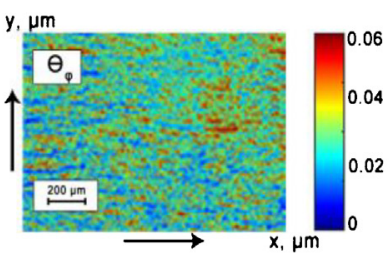

(3)

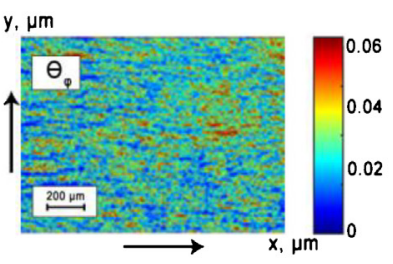

(7)

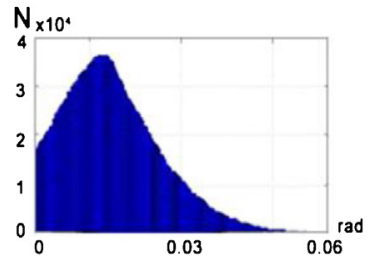

(4)

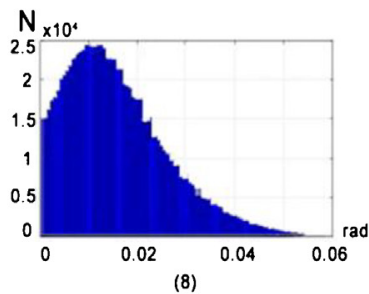

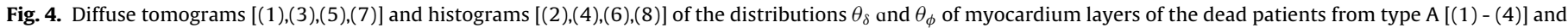
type B $[(5)-(8)]$.

Table 1

Central moments of polarization-phase tomograms distributions $T(x, y)$ of histological sections of the myocardium.

\begin{tabular}{|c|c|c|c|c|c|c|c|c|}
\hline \multirow{2}{*}{$Z_{i=1 ; 2 ; 3 ; 4}$} & \multicolumn{2}{|l|}{$\mathrm{T}(\delta)$} & \multicolumn{2}{|l|}{$\mathrm{T}(\varphi)$} & \multicolumn{2}{|l|}{$\mathrm{T}(\tau)$} & \multicolumn{2}{|l|}{$\mathrm{T}(\chi)$} \\
\hline & Type A & Type B & Type A & Type B & Type A & Type B & Type A & Type B \\
\hline$Z_{1} \times 10^{-1}$ & $\begin{array}{l}0.11 \pm \\
0.009\end{array}$ & $\begin{array}{l}0.07 \pm \\
0.005\end{array}$ & $\begin{array}{l}0.085 \pm \\
0.006\end{array}$ & $\begin{array}{l}0.076 \pm \\
0.005\end{array}$ & $\begin{array}{l}0.09 \pm \\
0.007\end{array}$ & $\begin{array}{l}0.05 \pm \\
0.004\end{array}$ & $\begin{array}{l}0.075 \pm \\
0.005\end{array}$ & $\begin{array}{l}0.067 \pm \\
0.004\end{array}$ \\
\hline$Z_{2} \times 10^{-1}$ & $\begin{array}{l}0.15 \pm \\
0.011\end{array}$ & $\begin{array}{l}0.09 \pm \\
0.006\end{array}$ & $\begin{array}{l}0.13 \pm \\
0.01\end{array}$ & $\begin{array}{l}0.11 \pm \\
0.0084\end{array}$ & $\begin{array}{l}0.11 \pm \\
0.0081\end{array}$ & $\begin{array}{l}0.08 \pm \\
0.005\end{array}$ & $\begin{array}{l}0.11 \pm \\
0.009\end{array}$ & $\begin{array}{l}0.096 \pm \\
0.0074\end{array}$ \\
\hline$Z_{3}$ & $\begin{array}{l}0.87 \pm \\
0.062\end{array}$ & $\begin{array}{l}0.51 \pm \\
0.039\end{array}$ & $\begin{array}{l}1.07 \pm \\
0.088\end{array}$ & $\begin{array}{l}0.89 \pm \\
0.065\end{array}$ & $\begin{array}{l}1.28 \pm \\
0.092\end{array}$ & $\begin{array}{l}0.91 \pm \\
0.0 .69\end{array}$ & $\begin{array}{l}1.33 \pm \\
0.108\end{array}$ & $\begin{array}{l}1.18 \pm \\
0.095\end{array}$ \\
\hline$Z_{4}$ & $\begin{array}{l}1.19 \pm \\
0.097\end{array}$ & $\begin{array}{l}0.68 \pm \\
0.042\end{array}$ & $\begin{array}{l}1.46 \pm \\
0.12\end{array}$ & $\begin{array}{l}1.18 \pm \\
0.098\end{array}$ & $\begin{array}{l}1.89 \pm \\
0.13\end{array}$ & $\begin{array}{l}1.06 \pm \\
0.092\end{array}$ & $\begin{array}{l}1.54 \pm \\
0.13\end{array}$ & $\begin{array}{l}1.31 \pm \\
0.11\end{array}$ \\
\hline
\end{tabular}

Table 2

Balanced accuracy of the method of polarization-phase tomography of myocardium layers.

\begin{tabular}{|l|l|l|l|l|l|}
\hline \multirow{5}{*}{$A c\left(Z_{i}\right)$} & $Z_{i=1 ; 2 ; 3 ; 4}$ & $\mathrm{~T}(\delta)$ & $\mathrm{T}(\varphi)$ & $\mathrm{T}(\tau)$ & $\mathrm{T}(\chi)$ \\
\cline { 2 - 6 } & $Z_{1}$ & $86 \%$ & $62 \%$ & $74 \%$ & $61 \%$ \\
\cline { 2 - 6 } & $Z_{2}$ & $88 \%$ & $65 \%$ & $68 \%$ & $65 \%$ \\
\cline { 2 - 6 } & $Z_{3}$ & $91 \%$ & $76 \%$ & $78 \%$ & $72 \%$ \\
\cline { 2 - 6 } & $Z_{4}$ & $95 \%$ & $78 \%$ & $80 \%$ & $69 \%$ \\
\hline
\end{tabular}

the increase in the average and dispersion of the corresponding distributions $N\left(\theta_{\phi}\right)$ and (Fig. 4, (6), (8)].

\subsection{Statistical approach in the differentiation of necrotic and} pathological changes in the optical anisotropy of biological tissues of human organs

To determine the quantitative criteria, according to which it is possible to reliably differentiate the necrotic (type A and type B) and pathological (type $C$ and type D) changes in the optical anisotropy of biological tissues of human organs, we used the technique developed in Refs. [33-35]. Here, within the sample groups, the histological sections of the myocardium and the connective tissue component of the vaginal wall determine the average $\left(\bar{Z}_{i=1 ; 2 ; 3 ; 4}\right)$ and standard deviations $( \pm 2 \psi)$ of the central moments $Z_{i=1 ; 2 ; 3 ; 4}$ (Tables 1 and 3). For each of these parameters we define the balanced accuracy (Ac - Tables 2 and 4), which characterizes the number of correct and incorrect estimates ( $\ddot{A}-B a ̈ n d ~ \ddot{C}-D)$ obtained by the polarization-phase (Tables 1 and 3 ) and diffuse (Tables 2 and 4) Mueller matrix tomography methods.
Table 3

Central moments of diffuse tomogram distributions $\tilde{T}(x, y)$ of histological sections of the myocardium.

\begin{tabular}{|l|l|l|l|l|}
\hline \multirow{2}{*}{$Z_{i=1 ; 2 ; 3 ; 4}$} & \multicolumn{2}{|l|}{$\widetilde{\mathrm{T}}\left(\theta_{\delta}\right)$} & $\widetilde{\mathrm{T}}\left(\theta_{p}\right)$ \\
\cline { 2 - 5 } & Type A & Type B & Type A & Type B \\
\hline \multirow{2}{*}{$Z_{1} \times 10^{-1}$} & $0.31 \pm$ & $0.44 \pm$ & $0.095 \pm$ & $0.11 \pm$ \\
& 0.019 & 0.025 & 0.006 & 0.007 \\
\hline \multirow{2}{*}{$Z_{2} \times 10^{-1}$} & $0.21 \pm$ & $0.34 \pm$ & $0.08 \pm$ & $0.095 \pm$ \\
& 0.011 & 0.016 & 0.005 & 0.007 \\
\hline \multirow{2}{*}{$Z_{3}$} & $1.25 \pm$ & $0.91 \pm$ & $0.81 \pm$ & $0.69 \pm$ \\
& 0.14 & 0.11 & 0.068 & 0.035 \\
\hline \multirow{2}{*}{$Z_{4}$} & $0.99 \pm$ & $0.73 \pm$ & $1.32 \pm$ & $1.02 \pm$ \\
& 0.061 & 0.042 & 0.15 & 0.12 \\
\hline
\end{tabular}

The obtained results (Table 1 ) of the intergroup statistical analysis of the aggregate of distributions $T(x, y)$ revealed the statistical reliability of the difference between the average values of the central moments $Z_{i=1 ; 2 ; 3 ; 4}$ within the two groups of samples of type $\mathrm{A}$ and type $B$ myocardium. 
Table 4

Balanced accuracy of the method of diffuse tomography of myocardium layers.

\begin{tabular}{|l|l|l|l|}
\hline \multirow{3}{*}{$A c\left(Z_{i}\right)$} & $Z_{i=1 ; 2 ; 3 ; 4}$ & $\tilde{\mathrm{T}}\left(\theta_{\delta}\right)$ & $\tilde{\mathrm{T}}\left(\theta_{\varphi}\right)$ \\
\cline { 2 - 4 } & $Z_{1}$ & $83 \%$ & $66 \%$ \\
\cline { 2 - 4 }$Z_{2}$ & $85 \%$ & $68 \%$ \\
\hline$Z_{3}$ & $75 \%$ & $62 \%$ \\
\hline$Z_{4}$ & $77 \%$ & $60 \%$ \\
\hline
\end{tabular}

It was observed that the parameters $Z_{i=3 ; 4}(\delta, \tau)$ appeared to be sensitive to the change in the structure of the tomograms $T(\delta)$ and $T(\tau)$ that represent the coordinate distributions of the birefringence and dichroism of fibrillar networks of myocardium samples (highlighted in gray in Table 1).

For the optical activity and dichroism of the polarization-phase tomograms $T(\phi)$ and $T(\chi)$ of myocardium histological sections are less informative. The difference between the values of $\tilde{Z}_{i=1 ; 2 ; 3 ; 4}$ in both groups of myocardium samples is not so vivid.

The data presented in Table 2 indicate high accuracy, according to the criteria of evidence-based medicine [35], in the differentiation of necrotic conditions of the myocardium by the method of polarization-phase tomography of the linear birefringence (Ac $\left(Z_{3 ; 4}(\delta)\right)>90 \%$ - excellent quality) of its fibrillar networks.

The accuracy of this method for the parameters of circular birefringence $(\phi)$ and optically anisotropic absorption $(\tau ; \quad \chi)$ does not exceed the satisfactory level $-A c\left(Z_{i=1-4}\right)<80 \%$.

The results of the statistical analysis of the coordinate structure of diffuse tomograms of phase anisotropy of both groups of myocardial samples are presented in Table 3.

The analysis of the obtained data proved that the statistical moments of the 1 st and 2nd orders, which characterize the average and the dispersion of distributions $N\left(\theta_{\phi}\right)$ and $N\left(\theta_{\phi}\right)$ (highlighted in gray), are the most sensitive statistical parameters of fluctuations $\theta_{\delta}$ and $\theta_{\phi}$. The greatest differences between the values $Z_{1}$ (1.42 times) and $Z_{2}$ (1.61 times) occur for diffuse tomograms $\tilde{T}\left(\theta_{\delta}\right)$ of the myocardium samples of the A and B types. For tomograms of the fluctuations in the magnitude of the circular birefringence $\tilde{T}\left(\theta_{\phi}\right)$ the differences between the statistical parameters showed the decrease of $Z_{1}$ (by 1.16 times) and $Z_{2}$ (by 1.19 times).

Table 4 presents the values of the balanced accuracy of the diffuse Mueller-matrix tomography method of the polycrystalline structure of the A and B types of myocardium depolarizing layers.

The data presented in Table 4 demonstrate a somewhat lower (by $7 \%-10 \%$ ) level of accuracy of the diffuse tomography method in comparison with the results of polarization-phase tomography (Table 2). Despite this, for distributions $\tilde{T}\left(\theta_{\delta}\right)$ indicate a good level of accuracy in the differentiation of histological sections of the A and B types of myocardium - Ac $\left(Z_{1 ; 2}\left(\theta_{\delta}\right)\right) \sim 83 \%-85 \%$.

\subsection{Potentiality of clinical application}

Table 5 presents the parameters of information value of method of polarization-phase and diffuse tomography of optical anisotropy of depolarizing ( $\Lambda=38 \% \div 47 \%$ ) histological sections of healthy tissue of the vaginal wall and of that with genitals prolapse.

As in the expert evaluation of degenerative-dystrophic changes in myocardial tissue, the accuracy of the clinical application of this methods of polarization-phase and diffuse Mueller matrix tomography in differential diagnosis of the connective tissue component of the vaginal wall at genital prolapse reaches the good (85\%-90\%) and high (>90\%) quality (highlighted in gray).

The results obtained are optimistic for the further development of the objective and rather fast ( $t \leq 15$ minutes) polarization methods for the clinical differentiation of the histological sections of strongly depolarizing biological tissues $-\Lambda=50 \% \div 90 \%$.

\section{Conclusions}

The theoretically substantiated and experimentally realized technique of polarization-phase and diffuse Mueller-matrix tomography in the differentiation of necrotic (myocardium) and pathological (vaginal wall) changes in birefringence and dichroism of partially depolarizing layers of biological tissues of human organs is proposed.

The parameters (central statistical moments of all orders) that characterize the structure of the polarization-phase and diffuse tomograms of histological sections of the myocardium (type A and type $B$ ), as well as the vaginal wall (type $C$ and type $D$ ) are the most sensitive to the changes in the phase and amplitude anisotropy.

High accuracy of differential diagnostics of the following is achieved:

- severity of necrotic changes in the myocardium (excellent quality);

- pathology of the vaginal wall with prolapse of the genitals (good quality).

\section{References}

[1] V.V. Tuchin, Tissue Optics: Light Scattering Methods and Instruments for Medical Diagnosis, vol. 166, second edition, SPIE Press, Bellingham, WA, PM, 2007.

[2] S. Bickel, W.M. Bailey, Stokes vectors, Mueller matrices, and polarization of scattered light, Am. J. Phys. 53 (1985) 468-478.

[3] X. Wang, G. Yao, L.H. Wang, Monte Carlo model and single-scattering approximation of polarized light propagation in turbid media containing glucose, Appl. Opt. 41 (2002) 792-801.

[4] X. Wang, L.-H. Wang, Propagation of polarized light in birefringent turbid media: a Monte Carlo study, J. Biomed. Opt. 7 (2002) 279-290.

[5] O.V. Angelsky, A.Ya Bekshaev, P.P. Maksimyak, A.P. Maksimyak, S.G. Hanson, Measurement of small light absorption in microparticles by means of optically induced rotation, Opt. Express 23 (6) (2015) 7152-7163.

[6] O.V. Angelsky, R.N. Besaha, A.I. Mokhun, I.I. Mokhun, M.O. Sopin, M.S. Soskin, Singularities in vectoral fields, Proc. SPIE. Int. Soc. Opt. Eng. 3904 (1999) 40-54.

[7] V.K. Polyanskii, O.V. Angelsky, P.V. Polyanskii, Scattering-induced spectral changes as a singular optical effect, Opt. Appl. 32 (4) (2002) 843-848.

Table 5

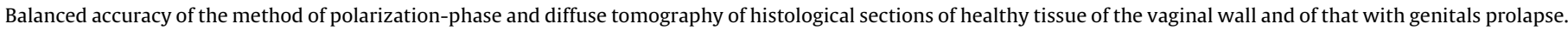

\begin{tabular}{|l|l|l|l|l|l|}
\hline & $Z_{i=1 ; 2 ; 3 ; 4}$ & $\mathrm{~T}(\delta)$ & $\mathrm{T}(\tau)$ & $\widetilde{\mathrm{T}}\left(\theta_{s}\right)$ & $\widetilde{\mathrm{T}}\left(\theta_{\varphi}\right)$ \\
\cline { 2 - 6 }$A c\left(Z_{i}\right)$ & $Z_{1}$ & $81 \%$ & $73 \%$ & $87 \%$ & $80 \%$ \\
\cline { 2 - 6 } & $Z_{2}$ & $82 \%$ & $68 \%$ & $84 \%$ & $88 \%$ \\
\cline { 2 - 6 } & $Z_{3}$ & $87 \%$ & $85 \%$ & $79 \%$ & $66 \%$ \\
\cline { 2 - 6 } & $Z_{4}$ & $91 \%$ & $82 \%$ & $85 \%$ & $64 \%$ \\
\hline
\end{tabular}


[8] O.V. Angelsky, M.P. Gorsky, S.G. Hanson, V.P. Lukin, I.I. Mokhun, P.V. Polyanskii, P.A. Ryabiy, Optical correlation algorithm for reconstructing phase skeleton of complex optical fields for solving the phase problem, Opt. Exp. 22 (5) (2014) 6186-6193.

[9] V.V. Tuchin, L. Wang, D.A. Zimnyakov, Optical Polarization in Biomedical Applications, New York, USA, 2006.

[10] R.A. Chipman, in: M. Bass (Ed.), Polarimetry in Handbook of Optics: Vol. IGeometrical and Physical Optics, Polarized Light, Components and Instruments, McGraw-Hill Professional, New York, 2010, pp. 22.1-22.37.

[11] N. Ghosh, M.F.G. Wood, I.A. Vitkin, in: V.V. Tuchin (Ed.), Polarized Light Assessment of Complex Turbid media Such as Biological Tissues via Mueller Matrix Decomposition in Handbook of Photonics for Biomedical Science, CRC Press, Taylor \& Francis Group, London, 2010, pp. 253-282.

[12] S.L. Jacques, in: D. Boas, C. Pitris, N. Ramanujam (Eds.), Polarized Light Imaging of Biological Tissues in Handbook of Biomedical Optics, CRC Press, Boca Raton, London, New York, 2011, pp. 649-669.

[13] A.G. Ushenko, V.P. Pishak, Laser polarimetry of biological tissue: principles and applications, handbook of coherent-domain optical methods, Biomed. Diagn. Environ. Mater. Sci. (2004) 239-321.

[14] O.V. Angelsky, A.G. Ushenko, Yu.A. Ushenko, V.P. Pishak, A.P. Peresunko, Statistical, correlation and topological approaches in diagnostics of the structure and physiological state of birefringent biological tissues, Handb. Photon. Biomed. Sci. (2010) 283-322.

[15] Yu.A. Ushenko, T.M. Boychuk, V.T. Bachynsky, O.P. Mincer, Diagnostics of Structure and Physiological State of Birefringent Biological Tissues: Statistical, Correlation and Topological Approaches, Handbook of Coherent-Domain Optical Methods, Springer Science+Business Media, 2013, 107.

[16] O.V. Angelsky, Y.Y. Tomka, A.G. Ushenko, Y.G. Ushenko, S.B. Yermolenko, 2-D tomography of biotissue images in pre-clinic diagnostics of their pre-cancer states, Proc. SPIE. Int. Soc. Opt. Eng. 5972 (2005) 158-162.

[17] Yu.A. Ushenko, V.A. Ushenko, A.V. Dubolazov, V.O. Balanetskaya, N.I. Zabolotna, Mueller-matrix diagnostics of optical properties of polycrystalline networks of human blood plasma, Opt. Spectrosc. 112 (2012) 884-892.

[18] V.A. Ushenko, O.V. Dubolazov, A.O. Karachevtsev, Two wavelength Mueller matrix reconstruction of blood plasma films polycrystalline structure in diagnostics of breast cancer, Appl. Opt. 53 (2014) B128-B139.

[19] Y.A. Ushenko, G.D. Koval, A.G. Ushenko, O.V. Dubolazov, V.A. Ushenko, O.Yu Novakovskaia, Mueller-matrix of laser-induced autofluorescence of polycrystalline films of dried peritoneal fluid in diagnostics of endometriosis, J. Biomed. Opt. 21 (7) (2016), 071116.
[20] N. Ortega-Quijano, J.L. Arce-Diego, Mueller matrix differential decomposition, Chin. Opt. Lett. 36 (2011) 1942-1944.

[21] N. Ortega-Quijano, J.L. Arce-Diego, Depolarizing differential Mueller matrices, Chin. Opt. Lett. 36 (2011) 2429-2431.

[22] V. Devlaminck, Physical model of differential Mueller matrix for depolarizing uniform media, J. Opt. Soc. Am. 30 (2013) 2196-2204.

[23] R. Ossikovski, V. Devlaminck, General criterion for the physical reliability of the differential Mueller matrix, Opt. Lett. 39 (2014) 1216-1219.

[24] V. Devlaminck, R. Ossikovski, Uniqueness of the differential Mueller matrix of uniform homogeneous media, Opt. Lett. 39 (2014) 3149-3152.

[25] R. Ossikovski, O. Arteaga, Statistical meaning of the differential Mueller matrix of depolarizing homogeneous media, Opt. Lett. 39 (2014) 4470-4473.

[26] R. Ossikovski, Differential matrix formalism for depolarizing anisotropic media, Chin. Opt. Lett. 36 (2011) 2330-2332

[27] A.G. Ushenko, A.V. Dubolazov, V.A. Ushenko, O.Y. Novakovskaya, Statistical analysis of polarization-inhomogeneous fourier spectra of laser radiation scattered by human skin in the tasks of differentiation of benign and malignant formations, J. Biomed. Opt. 000121 (7) (2016), 071110.

[28] V.A. Ushenko, N.D. Pavlyukovich, L. Trifonyuk, Spatial-frequency azimuthally stable cartography of biological polycrystalline networks, Int. J. Opt. 2013 (2013), 683174.

[29] V.P. Prysyazhnyuk, Yu.A. Ushenko, A.V. Dubolazov, A.G. Ushenko, V.A. Ushenko, Polarization-dependent laser autofluorescence of the polycrystalline networks of blood plasma films in the task of liver pathology differentiation, Appl. Opt. 55 (2016) B126-B132.

[30] O.V. Angelsky, S.G. Hanson, P.P. Maksimyak, A.P. Maksimyak, C.Yu Zenkova, P.V. Polyanskii, D.I. Ivanskyi, Influence of evanescent wave on birefringent microplates, Opt. Exp. 25 (2017) 2299-2311.

[31] O.V. Angelsky, A.Ya Bekshaev, P.P. Maksimyak, A.P. Maksimyak, S.G. Hanson, C.Yu Zenkova, Self-diffraction of continuous laser radiation in a disperse medium with absorbing particles, Opt. Exp. 21 (7) (2013) 8922-8938.

[32] Yu. Ushenko, V. Bachynsky, O. Vanchulyak, A. Dubolazov, M. Garazdyuk, V. Ushenko, Jones-matrix mapping of complex degree of mutual anisotropy of birefringent protein networks during the differentiation of myocardium necrotic changes, Appl. Opt. 55 (2016) B113-B119.

[33] L.D. Cassidy, Basic concepts of statistical analysis for surgical research, J. Surg. Res. 128 (2005) 199-206.

[34] C.S. Davis, Statistical Methods of the Analysis of Repeated Measurements, Springer-Verlag, New York, 2002

[35] A. Petrie, B. Sabin, Medical Statistics at a Glance, Blackwell Publishing, London, 2005. 